\title{
Optimal Strategy of the Patient-Driven Command Controller for Restoring Hand Functions by Functional Electrical Stimulation
}

\author{
Y. H. Chiou' ${ }^{1}$, J. J. Luh ${ }^{2}$, S. S. Chen ${ }^{3}$, J. S. Lai ${ }^{2}$, T. S. Kuo ${ }^{1,4}$ \\ ${ }^{1}$ Department of Electrical Engineering, National Taiwan University, Taiwan, R.O.C. \\ ${ }^{2}$ Department of Physical Medicine and Rehabilitation, National Taiwan University Hospital, Taiwan, R.O.C. \\ ${ }^{3}$ Department of Physical Medicine and Rehabilitation, Taipei Medical University Hospital, Taiwan, R.O.C. \\ ${ }^{4}$ Graduate Institute of Biomedical Engineering, National Taiwan University, Taipei, Taiwan, R.O.C.
}

\begin{abstract}
This study is about the optimal strategy of restoring hand functions of the quadriplegics by functional electrical stimulation (FES). Nowadays, there are many assistive devices developed for restoring the quadriplegics' lost functions by FES. If patients can use their residual capabilities to control these devices, it will be very exciting thing for them. However, the input sources or the command controller are usually complicated or inconvenient for them to use. If there is the optimal strategy of controlling built, the quadriplegies will benefit much from it. In this study, we had designed the optimal strategy of the patient-driven command controller for restoring their lost hand functions by FES. The proposed optimal control strategy can also be modified and applied for different rehabilitation applications.
\end{abstract}

Keywords - Optimal Strategy, Command Controller, Hand Function, Quadriplegics, Functional Electrical Stimulation

\section{INTRODUCTION}

Quadriplegics, with hand paralysis who were due to spinal cord injuries (SCI) or other neurological impairments, lost their ability to grasp objects for activities of daily life (ADL), such as eating, drinking, writing, brushing teeth, etc. They are unable to perform these activities independently, and it may also increase the loading to their families both economically and mentally. Apparently, it is very desirable and urgent to develop a practical system that can be used by the quadriplegics to restore their hand functions.

There are many assistive devices developed for restoring the lost functions of quadriplegics in the recent decade [1-5]. Functional electrical stimulation (FES) and neural prostheses are very popular in clinic applications. Although these systems are highly developed, the input sources or command controller are very complicated and inconvenient for the patients to use. If there is existed an optimal strategy for the input source or command controller, it will be very helpful for them to restore their lost functions. Besides, from the aspects of rehabilitation and psychology, they will benefit more if they could use their residual capabilities to control these assistive devices. In this study, we will have discussions on the optimal strategy of the patient-driven command controller in rehabilitation applications, and we will take the restoration of hand functions by functional electrical stimulation for example.

\section{METHODOLOGY}

It is very important to design the optimal strategy for restoring hand functions by FES. In order to find the optimal strategy of different input sources before the paralyzed hand muscles can be actually controlled by a command controller, there are several essential issues need to be dealt with.

Firstly, it is necessary to systemically profile the muscle groups that perform hand functions when applying the electrical stimulation for individuals. These parameters include threshold, saturation, spillover, recruitment curves of position and force.

Secondly, since each individual will have different stimulating parameters, we need to develop a systematic procedure and related devices to identify them. These parameters could be as a base on the development of stimulating template. Combining with FES, the stimulating template can be tuned to get the best available grasp function. Besides, we also need to synthesize the stimulation template to get the so-called multi-state setting of the hand motion. To analyze the hand motions, there are multi-state settings such as the neural position of the hand, hand opening, grasping, etc.

Thirdly, in order to find proper electrical stimulation parameters during different hand motions, there needs the optimal stimulation template. After finding the profiles of targeted muscle groups and the proper electrical stimulation parameters, the motions would be produced by FES more smoothly. On the other hand, the aim of this study is to develop a patient-driven command controller for the quadriplegics, too. With their residual capabilities, they can control the assistive devices better using this optimal strategy. The flowchart of optimal strategy for FES is showed in Fig. 1.

To begin with this study, we will have normal people to build the electrical stimulating templates and muscle profiling system. When they have been done well and also have some preliminary results, the quadriplegics, such as the $\mathrm{C} 5 / \mathrm{C} 6$ patients, will be involved in the following study. In the next several parts, there are some results and discussions of the optimal strategy design for FES. 


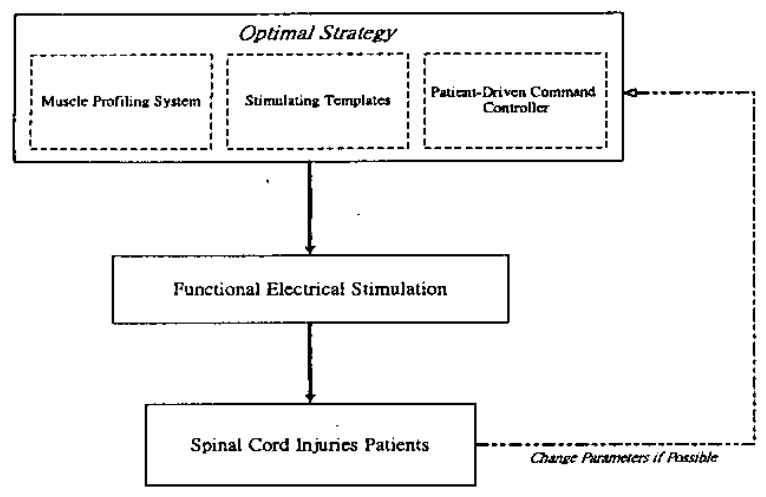

Fig. 1. Optimal strategy for functional electrical stimulation system

\section{RESULTS}

In this study, we want to develop the optimal strategy of FES for restoring quadriplegics' hand functions (such as grasping or releasing). Beginning the design of optimal strategy for functional electrical stimulation, we should know the states of hand motions. The cylindrical grasping and lateral pinching were selected in this experiment. The motion-oriented modules had been designed to get the profiles of related muscle groups, as illustrated in Fig. 2. The muscles included flexor digitorum superficialis, flexor digitorum profundus, thenar muscle, extensor digitorum, extensor pollicis longus, and extensor pollicis brevis. The numbers of stimulating channels were three, and we had the electrical stimulating sequences as depicted in Fig. 3. Besides, the electrical stimulator used here was developed by our own group [6], and it also had user-friendly humanmachine interface designed by LabVIEW program [7]. Three able-bodied subjects were participated in this study. The preliminary results of the motion-oriented modules were showed in Table I.

\section{DISCUSSION \& CONCLUSION}

The optimal strategy is surely important for the patientdriven command controller. Patients can use their residual capabilities to control the assistive devices elegantly if the optimal control strategy is applied. In this paper, we hope that with the designed command controller, the patients can voluntarily control the hand motions and grasping forces by themselves. Indeed, it is expected that the command controller should be able to (1) detect and quantify the user's residual capabilities and (2) calibrate it to match the hand's physical motions and forces in real time.
However, due to limitations of electrode types, number of electrodes, the optimal approaching trajectory, grasping posture, and grasping force for a normal subject may not be available in a quadriplegic. Thus, how to systematically synthesize and tune the stimulation template to get best available grasp function will be another important issue. There must have several electrical stimulation templates existed that can match the desired hand motions and grasp forces properly. Therefore, we would like to find the ways to optimize the stimulation templates and to get the optimal control strategy as possible. The optimal control strategy would include having fewer electrodes to get the best results by electrical stimulation, having the more natural way to control FES, and producing more desired hand motions as possible.

In this paper, we had showed the methods to find the targeted muscle profiles and to create the electrical stimulation templates. Although we just had normal subjects to test our algorithms, we will arrange the SCI patients for the next study. It really has the importance to find the optimal strategy of patient-driven command controller for restoring hand functions by function electrical stimulation. We will have more research and work done in the near future.

\section{ACKNOWLEDGMENT}

The authors acknowledge the financial support of the National Science Council of Republic of China under grant NSC 91-2614-E-038-001.

\section{REFERENCES}

[1] C. A. Wijman, K. C. Strch, C. L. Van Doren, G. B. Thrope, P. H. Peckham, and M. W. Keith, "Functional evaluation of quadriplegic patients using a hand neuroprosthesis", Arch. Phys. Med. Rehabil., pp. 1053-7, 1990.

[2] R. H. Nathan, "Control strategies in FNS systems for the upper extremities", Crit. Rev. Biomed. Eng., pp. 485-568, 1993.

[3] J. C. Burelbach, and P. E. Crago, "Instrumented assessment of FNS hand control durìg specific manipulation tasks," IEEE Trans. Rehab. Eng., pp. 165-176, 1994.

[4] T. R. D. Scoot, P. H. Peckham, and K. L. Kilgore, "Tri-state myoelectric control of bilateral upper extremity neuroprostheses for tetraplegic individuals," IEEE Trans. Rehab. Eng., pp. 251$263,1996$.

[5] J. H. Grill, and P. H. Peckham, "Functional neuromuscular stimulation for combined control of elbow extension and hand grasp in C5 and C6 quadriplegics," IEEE Trans. Rehab. Eng., pp. 190-199, 1998.

[6] H. C. Wu, S. T. Yang, and T. S. Kuo, "A versatile multichannel direct-synthesized electrical stimulator for FES applications," IEEE Trans. Instrum. Meas., vol. 51, no. 1, pp. 2-9, Feb. 2002.

[7] Y. H. Chiou, R. S. Tzeng, J. S. Lai, and T. S. Kuo, "A digital signal processor based functional electrical stimulation system with its user interface design," in Proc. 2nd Joint EMBS-BMES Conf., Houston, TA, pp. 352, 2002. 
TABLE I

THE MAXIMUN ELECTRICAL STIMULATION CURRENTS FOR STIMULATING TEMPLATES DESIGNS

\begin{tabular}{lcccc}
\hline & Opening & Grasping & Frequency & Pulsewidth \\
\hline Subject 1 & $17 \mathrm{~mA}$ & $25 \mathrm{~mA}$ & $25 \mathrm{~Hz}$ & $300 \mathrm{us}$ \\
Subject 2 & $15 \mathrm{~mA}$ & $20 \mathrm{~mA}$ & $25 \mathrm{~Hz}$ & $300 \mathrm{us}$ \\
& & & & \\
Subject 3 & $20 \mathrm{~mA}$ & $26 \mathrm{~mA}$ & $25 \mathrm{~Hz}$ & 300 us \\
\hline
\end{tabular}

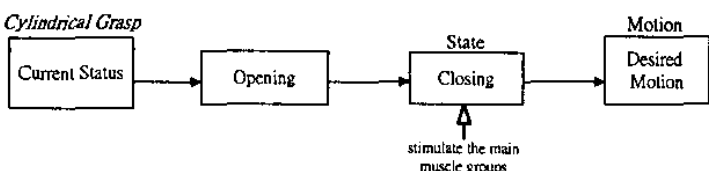

(a)

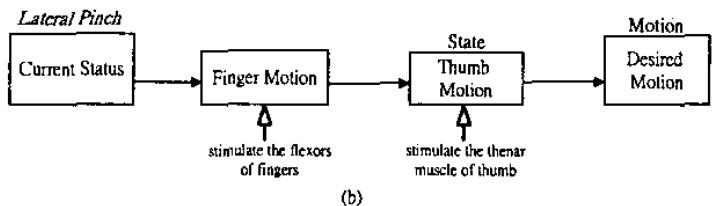

Fig. 2. (a) Motion-oriented module of cylindrical grasping,

(b) Motion-oriented module of lateral pinching

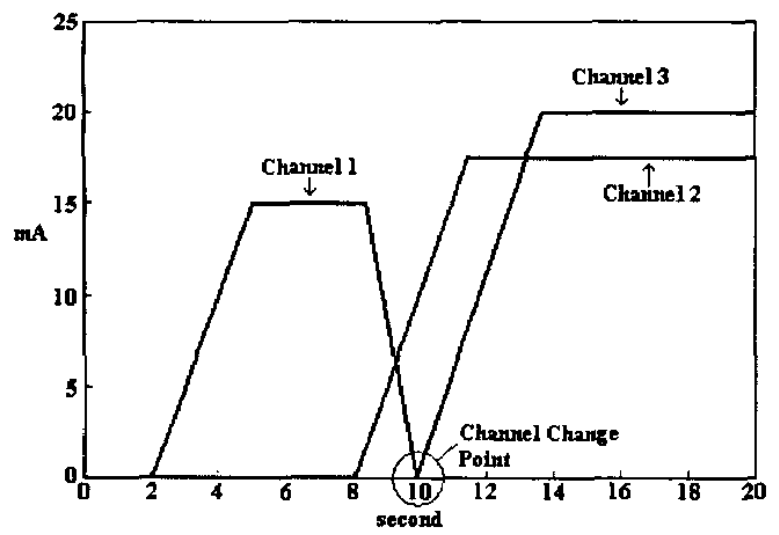

Fig. 3. Electrical stimulating sequences for three channels 